OPEN ACCESS

Edited by:

Jong-Min Kim,

Seoul National University Bundang

Hospital, South Korea

Reviewed by:

Daniele Nosi,

University of Florence, Italy Jacob Raber

Oregon Health and Science University, United States

*Correspondence:

Dmytro Shepilov

shepilov@biph.kiev.ua

tORCID:

Dmytro Shepilov

orcid.org/0000-0002-5698-1210

Nittaya Marungruang

orcid.org/0000-0001-9769-7980

Galyna Ushakova orcid.org/0000-0002-5633-2739

Diana Muraviova orcid.org/0000-0001-8382-5857

Frida Hållenius orcid.org/0000-0001-8203-9635

Olena Prykhodko orcid.org/0000-0002-5788-9176

Galyna Skibo orcid.org/0000-0003-2187-6178

Specialty section:

This article was submitted to Neuroenergetics, Nutrition and Brain Health,

a section of the journal Frontiers in Nutrition

Received: 23 May 2020 Accepted: 11 January 2022 Published: 16 February 2022

Citation:

Shepilov D, Kovalenko T, Osadchenko I, Smozhanyk K Marungruang N, Ushakova $G$ Muraviova D, Hållenius F, Prykhodko O and Skibo G (2022) Varying Dietary Component Ratios and Lingonberry Supplementation May Affect the Hippocampal Structure of ApoE-/-

Mice. Front. Nutr. 9:565051. doi: 10.3389/fnut.2022.56505

\section{Varying Dietary Component Ratios and Lingonberry Supplementation May Affect the Hippocampal Structure of ApoE-/- Mice}

\author{
Dmytro Shepilov ${ }^{1 *}$, Tatiana Kovalenko ${ }^{1}$, Iryna Osadchenko ${ }^{1}$, Kateryna Smozhanyk ${ }^{1}$, \\ Nittaya Marungruang ${ }^{2 \dagger}$, Galyna Ushakova ${ }^{3+}$, Diana Muraviova ${ }^{3 \dagger}$, Frida Hållenius ${ }^{2 \dagger}$, \\ Olena Prykhodko ${ }^{2 \dagger}$ and Galyna Skibo ${ }^{1 \dagger}$ \\ ${ }^{1}$ Department of Cytology, Bogomoletz Institute of Physiology, Kyiv, Ukraine, ${ }^{2}$ Department of Food Technology, Engineering \\ and Nutrition, Lund University, Lund, Sweden, ${ }^{3}$ Department of Biochemistry and Physiology, Oles Honchar Dnipro National \\ University, Dnipro, Ukraine
}

Objective: This study aimed to investigate and compare the morphological and biochemical characteristics of the hippocampus and the spatial memory of young adult ApoE-/- mice on a standard chow diet, a low-fat diet (LFD), a high-fat diet (HFD), and an HFD supplemented with lingonberries.

Methods: Eight-week-old ApoE-/- males were divided into five groups fed standard chow (Control), an LFD (LF), an HFD (HF), and an HFD supplemented with whole lingonberries $(H F+W h L B)$ or the insoluble fraction of lingonberries $(H F+I n s L B)$ for 8 weeks. The hippocampal cellular structure was evaluated using light microscopy and immunohistochemistry; biochemical analysis and T-maze test were also performed. Structural synaptic plasticity was assessed using electron microscopy.

Results: ApoE-/- mice fed an LFD expressed a reduction in the number of intact CA1 pyramidal neurons compared with HF+InsLB animals and the 1.6-3.8-fold higher density of hyperchromic (damaged) hippocampal neurons relative to other groups. The LF group had also morphological and biochemical indications of astrogliosis. Meanwhile, both LFD- and HFD-fed mice demonstrated moderate microglial activation and a decline in synaptic density. The consumption of lingonberry supplements significantly reduced the microglia cell area, elevated the total number of synapses and multiple synapses, and increased postsynaptic density length in the hippocampus of ApoE-/- mice, as compared to an LFD and an HFD without lingonberries.

Conclusion: Our results suggest that, in contrast to the inclusion of fats in a diet, increased starch amount (an LFD) and reduction of dietary fiber (an LFD/HFD) might be unfavorable for the hippocampal structure of young adult (16-week-old) male ApoE-/mice. Lingonberries and their insoluble fraction seem to provide a neuroprotective effect on altered synaptic plasticity in ApoE-/- animals. Observed morphological changes in the hippocampus did not result in notable spatial memory decline.

Keywords: hippocampus, lingonberries, dietary fiber, glial cells, ApoE-/- mice, low- and high-fat diets, starch, structural synaptic plasticity 


\section{INTRODUCTION}

Apolipoprotein E (ApoE) is a protein that binds and transports cholesterol throughout the body, particularly in the CNS and liver $(1,2)$. The protein plays a crucial role in maintaining neuronal functions, acting as a trophic, antioxidant factor and a mediator of the immune response during brain development and in response to cerebral damage (3). Additionally, ApoE is involved in debris clearance from brain tissues and the stimulation of nerve cell regeneration under normal and some pathological states, including aging and neurodegenerative diseases (4).

The ApoE-knockout mouse model (ApoE-/-) is widely used to study the consequences of ApoE deficiency (5). ApoE-/- mice demonstrate lipid metabolism deviations, such as hypercholesterolemia, and elicit atherosclerotic lesion formation with further inflammation and degradation of the extracellular matrix, especially with age (6). Reportedly, the plasma cholesterol level in ApoE-/- mice is 5- to 10-fold higher than in wildtype mice on a high-fat diet (HFD) (7). There is also evidence that ApoE-/- mice have abnormal tau protein phosphorylation, cholinergic dysfunction, synaptic loss, as well as memory and antioxidant metabolism impairments, which may negatively impact the function and integrity of the nervous system $(8,9)$.

The loss of synaptophysin-positive nerve terminals and MAP2-positive dendrites in the neocortex and hippocampus of ApoE-deficient mice on a standard diet has been detected using immunohistochemistry. There have also been reports on a drastic decrease in $\alpha$ - and $\beta$-tubulin immunoreactivity in ApoE-/- mice that might indicate impaired intracellular transport. In addition, ApoE-deficient mice tend to have an increased activation level of microglia (10). ApoE-/- mice, after HFD feeding, have been shown to exhibit cell swelling, nuclear hyperchromatosis, and karyopiknosis of the pyramidal neurons, as well as lipid accumulation in the hippocampus. Furthermore, the increased expression of several proteins linked to apoptosis activation (PCSK9, BACE1, caspase-3, and Bax) has been observed in CA3 neurons (11). Another study revealed that ApoE deficiency enhances the risk of hippocampal oxidative damage, while an HFD deteriorates oxidation (12). Also, high-cholesterol diets affect microglia by increasing the formation of inflammatory mediators (13). In contrast, caloric restriction in ApoE-/mice initiates neuroprotection manifested through the growing number of PSD95-positive neurons and better performance in Morris water maze (14), a learning and spatial memory test that has been shown to strongly correlate with hippocampal synaptic plasticity and function (15). Yet, some researchers have emphasized that a neuroinflammatory response with increased CD68+ cells in the CA1 area of ApoE-/- mice arises even on a standard rodent chow diet, and the level of inflammation remains unchanged when switching to an HFD (4).

Recently, the neuroprotective effects of berries have garnered increasing attention due to the reported antioxidant and anti-inflammatory properties derived from their bioactive components, such as dietary fiber and polyphenols (16). An extract of lingonberries, Vaccinium vitis-idaea, rich in dietary fiber and polyphenols, has been demonstrated to have a beneficial effect on cultured in vitro cardiomyocytes, neurons, and astrocytes $(17,18)$. Our prior study in 8-week-old ApoE-/mice showed that the inclusion of lingonberries or their insoluble fraction in an HFD (38\% of kcal from fat) feed for 8 weeks modified gut microbiota composition, which was associated with improvements in metabolic parameters, short-chain fatty acid profiles in serum, and increased hippocampal synaptic density (19).

To extend that study, the present research further explores the morphological and biochemical parameters of the hippocampus of young adult ApoE-/- mice after 8 weeks of feeding them an HFD containing whole lingonberries (HF+WhLB) or the insoluble fraction of lingonberries (HF+InsLB). We also investigated the cognitive function and hippocampal CA1 area morphology of ApoE-deficient mice on a standard chow, the lowfat diet (LFD), and an HFD. Our results may provide a better understanding of the effects of different levels of fats and other constituents in a diet on the structure of the hippocampus of ApoE-/- mice and lend further support to the potential benefit of bioactive components from berries, specifically lingonberries, on the brain.

\section{MATERIALS AND METHODS}

\section{Animals and Diets}

Male ApoE- $/$ - mice ( $n=49$; Taconic Biosciences Inc., Denmark) arrived at the animal facility at the age of 7 weeks $(21.9 \pm 0.2 \mathrm{~g})$ and were left to adapt to the environment $\left(22^{\circ} \mathrm{C}, 12 \mathrm{~h}\right.$ light-dark cycle) and diets for 1 week prior to starting the experiment. Following the adaptation period, animals were randomly allocated to 5 groups ( $n=9-10 /$ group $)$ based on mouse strain and nutrition: (1) control-ApoE-/- mice fed a standard rodent chow diet $(7.4 \%$ of kcal from fat, containing soybean oil) (RM1, SDS, UK); (2) LF ApoE-/- mice fed a customized LFD (12\% of kcal from fat, containing soybean oil) (Research Diets Inc., New Brunswick, USA); (3) HF ApoE-/- mice fed a customized HFD (38\% of kcal from fat, containing soybean oil, lard, and cholesterol) (Research Diets Inc., New Brunswick, USA); $(4,5) \mathrm{HF}+$ WhLB and HF+InsLB ApoE-/- mice fed a customized HFD with the inclusion of whole lingonberries or their water-insoluble fraction, respectively (Research Diets Inc., New Brunswick, USA) (Figure 1). Information about the diet composition is given in Table 1. A more detailed description of the diet preparation and inclusion of the berries is provided elsewhere (19). The experimental period lasted 8 weeks, at the end of which all the animals were 16 weeks old (commonly referred to as young adults). Duration of the feeding is based on our prior research on the bioactive effects of lingonberries (20). The investigation was carried out according to the principles in Directive 2010/63/EU of the European Union and approved by the local ethical committee at Lund University (Sweden).

\section{T-Maze Spontaneous Alternation Test}

The spatial memory of all mice was evaluated using the T-maze spontaneous alternation test 1 week before the end of the feeding period, i.e., after 7 weeks on the diets. The test was carried out per Deacon and Rawlins, with some modifications (21). Briefly, 


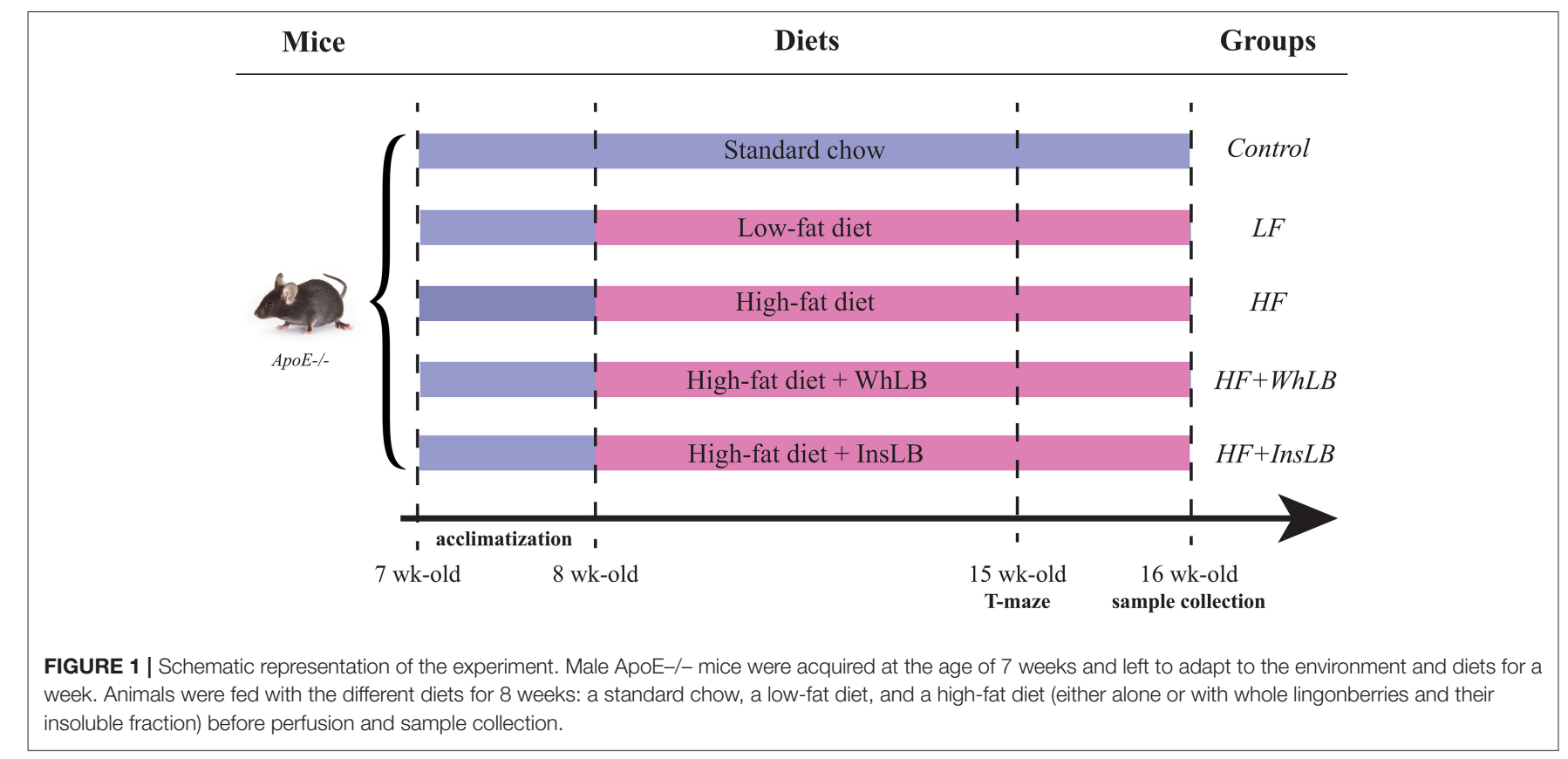

TABLE 1 | Composition of the experimental diets.

\begin{tabular}{|c|c|c|c|c|c|c|c|c|}
\hline \multirow[b]{2}{*}{ Nutrient } & \multicolumn{2}{|c|}{ LF } & \multicolumn{2}{|c|}{ HF } & \multicolumn{2}{|c|}{ HF + WhLB } & \multicolumn{2}{|c|}{ HF + InsLB } \\
\hline & gm $\%$ & kcal\% & gm $\%$ & kcal\% & gm $\%$ & kcal\% & gm $\%$ & kcal\% \\
\hline Protein & 15 & 16 & 15 & 14 & 15 & 14 & 15 & 14 \\
\hline Carbohydrate & 72 & 72 & 57 & 48 & 39 & 34 & 43 & 37 \\
\hline Fat & 5 & 12 & 18 & 38 & 18 & 38 & 18 & 38 \\
\hline Ingredient & gm & kcal & gm & kcal & gm & kcal & gm & kcal \\
\hline Casein & 157.5 & 630 & 157.5 & 630 & 157.5 & 630 & 157.5 & 630 \\
\hline Corn starch & 713.5 & 2,540 & 544.9 & 1,940 & 362.9 & 1,292 & 401.7 & 1,430 \\
\hline Sucrose & 50 & 200 & 50 & 200 & 50 & 200 & 50 & 200 \\
\hline Cellulose & 60 & 0 & 60 & 0 & 0 & 0 & 0 & 0 \\
\hline Lingonberries (whole berries) ${ }^{a}$ & 0 & 0 & 0 & 0 & 264.3 & $648^{c}$ & 0 & 0 \\
\hline Lingonberries (insoluble fraction) $)^{b}$ & 0 & 0 & 0 & 0 & 0 & 0 & 215.3 & $510^{c}$ \\
\hline Soybean oil & 50 & 450 & 50 & 450 & 50 & 450 & 50 & 450 \\
\hline Lard & 0 & 0 & 140 & 1,260 & 140 & 1,260 & 140 & 1,260 \\
\hline Cholesterol & 0 & 0 & 10 & 0 & 10 & 0 & 10 & 0 \\
\hline Mineral mix & 35 & 0 & 35 & 0 & 35 & 0 & 35 & 0 \\
\hline Vitamin mix & 10 & 40 & 10 & 40 & 10 & 40 & 10 & 40 \\
\hline Total & 1,076 & 3,860 & $1,057.4$ & 4,520 & 1,079.7 & 4,520 & $1,069.5$ & 4,520 \\
\hline
\end{tabular}

${ }^{a} 60 \mathrm{~g} / \mathrm{kg}(\mathrm{dwb})$ total dietary fiber.

${ }^{b} 60 \mathrm{~g} / \mathrm{kg}(\mathrm{dwb})$ insoluble dietary fiber.

${ }^{c}$ Calculated energy from carbohydrate content, excluding dietary fiber content in lingonberries or lingonberry insoluble fraction.

each mouse was carefully placed at the starting arm of the maze for $10 \mathrm{~s}$ before the animal was left to spontaneously choose one of the two arms (left or right; no rewarding system). Once the mouse chose the arm, the guillotine door was shut for $30 \mathrm{~s}$ to allow arm observation. The second trial was performed within $1 \mathrm{~min}$ of the first; the mouse was returned to the starting point and allowed to choose one of the two arms again spontaneously. Alternation, i.e., choosing the arm that was not visited before, was expected in the second trial. Three sets of trials a day (morning, afternoon, and evening hours) were performed for each mouse to avoid the influence of the daily cycle (19). The percentage of alternations and latency to choose (time spent to choose the arm) in the second trial were calculated.

\section{Sample Collection}

For morphological studies, 3 mice/group were anesthetized with isoflurane inhalation (Abbott Scandinavia AB, Sweden) and perfused transcardially with $0.1 \mathrm{M}$ phosphate buffer $+0.3 \%$ 
heparin $\left(+37^{\circ} \mathrm{C}\right)$, followed by perfusion with a fixative solution (2\% paraformaldehyde and $0.25 \%$ glutaraldehyde (SigmaAldrich, USA) in $0.1 \mathrm{M}$ phosphate buffer, $+4^{\circ} \mathrm{C}$ ). After perfusion, the brains were dissected and split into two hemispheres. The left hemispheres were used for immunohistochemistry, whereas the isolated hippocampi from the right hemispheres were examined under light and transmission electron microscopy. Coronal sections of the dorsal hippocampus were obtained from the anatomical areas between 1.65 and $2.48 \mathrm{~mm}$ posterior to the bregma, as defined by the Allen Mouse Brain Atlas (Figures 3A, 4A) (22). For biochemical assays, the remaining 5-6 animals/group were anesthetized with an overdose of pentobarbital (Nordvace, Sweden), after which their brains were quickly removed; both the left and right hippocampi were isolated on pre-chilled plates and immediately frozen until analysis.

\section{Immunohistochemistry}

Samples for immunohistochemistry were postfixed overnight in the same fixative as above at $+4^{\circ} \mathrm{C}$. 50 - $\mu \mathrm{m}$-thick sections of the hippocampus were obtained using a vibratome Leica VT1000A (Leica Biosystems, Germany). Free-floating sections were placed in wells of 24-well plates, rinsed with $0.1 \mathrm{M}$ phosphate buffer $(\mathrm{pH}$ 7,4 ), and treated with a blocking solution containing $1 \%$ BSA (Sigma-Aldrich, USA) and 0.3\% Triton X-100 (Sigma-Aldrich, USA) for $1 \mathrm{~h}$ at room temperature. Double immunofluorescent staining was then conducted. Polyclonal chicken antibodies against the glial fibrillary acidic protein (GFAP) (1:1500, Abcam, UK), a specific astrocyte marker, were used for astrocyte detection. Microglial cell identification was carried out with monoclonal rabbit antibodies against Iba-1 (1:750, WAKO, Japan) specifically expressed in microglia. Incubation with primary antibodies lasted $16 \mathrm{~h}$ at $+4^{\circ} \mathrm{C}$. After rinsing, sections were incubated with secondary antibodies; goat anti-chicken conjugated with Alexa Fluor 647 (1:1000, Invitrogen, USA) and donkey anti-rabbit conjugated with Alexa Fluor 488 (1:1000, Invitrogen, USA) for $1.5 \mathrm{~h}$, at room temperature, in the dark. The sections were then rinsed again, placed on histological slides, and mounted with Fluorescence Mounting Media (Dako, Denmark). $6-8$ sections per animal and 3-6 images $(220 \times 220 \mu \mathrm{m})$ from each section were analyzed. Images of hippocampal tissues were taken with an FV1000-BX61WI confocal microscope at 40x objective magnification (NA-0.65) (Olympus Corp., Japan).

\section{Light and Transmission Electron Microscopy}

The hippocampi were cut into $400-\mu \mathrm{m}$-thick transverse sections with a tissue chopper (McIlwain, UK). These sections were postfixed in $2.5 \%$ glutaraldehyde for $1.5 \mathrm{~h}$ and $1 \% \mathrm{OsO}_{4}$ for $1 \mathrm{~h}$ (23). Samples were then dehydrated in an ascending series of ethanol followed by dry acetone and embedded in EPON resin (Sigma-Aldrich, Switzerland) per standard protocol (24). The semi-thin $(1 \mu \mathrm{m})$ sections were produced with an LKB 8800 ultramicrotome (LKB, Sweden), stained with methylene blue, embedded in a Pertex mounting medium (HistoLab Products AB, Sweden), and visualized using an Olympus CX21 light microscope (Olympus Corp., Japan) (objective magnification-20x, NA-0.40). Ten to twelve semi-thin sections per animal and 2-3 images from each section were analyzed.

For electron microscopy, ultra-thin sections $(60-70 \mathrm{~nm})$ from the middle part of the CA1 stratum radiatum were stained with uranyl acetate and lead citrate and examined using a JEM-100CX transmission electron microscope (Jeol, Japan) at a magnification of $\times 10,000$.

\section{Morphometric Analysis}

ImageJ software, version 1.50i (NIH, USA), was used to study the hippocampal CA1 area structure of mice quantitatively. We counted the average number of intact and hyperchromic neurons per $1 \mathrm{~mm}$ length of a pyramidal layer (neuronal density) (25) and measured the density of GFAP-positive and Iba-1-positive cells per $1 \mathrm{~mm}^{2}$ of stratum pyramidale and stratum radiatum and the surface area of astrocytes/microglial cells. The number of synapses and their different types (simple, perforated and multiple types) per $100 \mu \mathrm{m}^{2}$ of stratum radiatum were defined. Simple and perforated synapses were considered synapses with continuous and discontinuous postsynaptic density, respectively. Synapses with more than one spine contacting the same presynaptic terminal were classified as multiple (Figure 5B). We also evaluated postsynaptic density (PSD) length.

\section{Biochemical Analysis (Enzyme-Linked Immunosorbent Assay)}

Hippocampal tissues were homogenized in a buffer solution consisting of $25 \mathrm{mM}$ Tris- $\mathrm{HCl}, 1 \mathrm{mM}$ EDTA, $2 \mathrm{mM} \beta$ dithiothreitol, $0.2 \mathrm{mM}$ PMSF, and $0.01 \%$ Merthiolate; $\mathrm{pH}$ 7,4 (100 mg of tissue/1 ml buffer) (23). Using differential ultracentrifugation, the water-soluble (containing the soluble forms of neural cell adhesion molecule-NCAM and glial fibrillary acidic protein-GFAP), membrane (containing the membrane form of NCAM), and cytoskeletal (containing the filamentous form of GFAP) protein fractions were obtained (26). We employed competitive ELISA to determine astrocyte- and neuron-specific proteins. Polyclonal rabbit primary antibodies against GFAP (Sigma, USA) and NCAM (Abnova, Germany), secondary anti-rabbit-IgG conjugated with HRP (Sigma, USA), and pure standard proteins (GFAP-Boehringer, Germany; NCAM-R\&D Systems, USA) were used. Measurements were performed at $492 \mathrm{~nm}$ with a spectrophotometer reader, Antos 2010 (Biochrom Ltd., Finland). Protein concentrations were expressed as microgram ( $\mu \mathrm{g})$ per $100 \mathrm{mg}$ of tissue.

\section{Statistical Analysis}

Statistical analysis was performed using GraphPad Prism, version 8.0.2 (GraphPad Software, Inc., USA). Categorical data from behavioral testing (spontaneous alternations) are expressed as percentages and were analyzed using Fisher's exact test. The normality distribution of continuous data was evaluated using the Shapiro-Wilk normality test, and the equality of variances was determined with Levene's test. Continuous behavioral data are given as median values, and interquartile ranges are presented in square brackets. These results are presented graphically as box plots: median values (central lines), interquartile ranges (25th-75th percentile, boxes), minimum 
and maximum (whiskers). Group comparisons were performed with the Kruskal-Wallis test, followed by Dunn's post hoc test. Morphological and biochemical results are presented as the mean \pm SEM. Raw data were assessed using one-way ANOVA, followed by Turkey's post hoc test. Pearson's correlation analysis was also conducted. $R$ values from 0 to \pm 0.29 , between \pm 0.3 and \pm 0.49 , and from \pm 0.5 to \pm 1 were consistent with a low, moderate, and high degree of correlation, respectively. Differences between compared groups were considered statistically significant when $p<0.05$.

\section{RESULTS}

\section{T-Maze Spontaneous Alternation}

LFD-fed mice produced the worst results in the T-maze spontaneous alternation test $(76.7 \%$ alternation) than animals fed other diets (86.7-93.3\%). However, there were no significant differences in the percentage of alternation (an indicator of spatial working memory; Figure 2A) and latency to choose (Figure 2B) between the experimental groups.

\section{The Density of CA1 Pyramidal Neurons}

Mice of the control, HF, HF+WhLB, and HF+InsLB groups were characterized by a normal architectonic organization of the hippocampal CA1 area. In those groups, pyramidal neurons had light nuclei with clear borders and visible 1-3 nucleoli, a narrow strip of normochromic cytoplasm around the nucleus, and well-defined descending apical dendrites oriented in the radial direction. Only a few pyramidal cells were hyperchromic. Structural disruptions in the CA1 area, in particular, the hyperchromatosis of the pyramidal cells, and the destruction of several apical dendrites were observed in semi-thin hippocampal sections of LFD-fed animals (Figure 3B). The average density of intact neurons in the control group was $136.3 \pm 5.3$ cells $/ \mathrm{mm}$ length of a pyramidal layer. The intact neuronal density in LFDfed mice was 13,16 , and $20 \%$ lower than in the control, HF, and $\mathrm{HF}+\mathrm{WhLB}$ groups, respectively (no significant differences), and $23 \%$ lower vs. the HF+InsLB group $(p<0.05)$ (Figure $3 \mathrm{C}$ ). Furthermore, there was a significant increase (by $264 \pm 16 \%$ ) in hyperchromic neuronal density in LFD-fed animals, compared to both control $(p<0.05)$ and HFD-fed mice $(p<0.05)$ (Figure 3D).

\section{Glial Cell Density and Surface Area}

The functional state of glial cells was determined using immunofluorescent microscopy based on their morphological characteristics. It is generally accepted that the reactive state of astrocytes includes the hypertrophy of their cell bodies and an increase in number, thickness, and length of primary processes, as against the resting state (27-29). As illustrated in Figures 4B,C, GFAP-positive cells in both the LF and HF+WhLB groups were characterized by the elongation and multiplication of base processes and the active spreading of side branches compared to other groups. In contrast, soma hypertrophy and the thickening of processes were displayed only in the hippocampi of LFDfed mice. The mentioned morphological alterations reflected the activation of the astrocyte component in these two groups. LFDfed ApoE-/- mice showed the highest density of GFAP-positive astrocytes in the hippocampal CA1 area $\left(456.6 \pm 24.7 \mathrm{cells} / \mathrm{mm}^{2}\right.$ of stratum pyramidale and stratum radiatum), which was $33 \pm 2 \%$ more than that of mice on standard chow and an HFD+InsLB $(p<0.05)$ and $23 \%$ more than that of mice on an HFD $(p=$ $0.09)$. There was also significant difference $(p<0.05)$ in the number of GFAP-positive cells between HF+WhLB-fed animals and their standard chow-fed counterparts (Figure 4D). As in the case of cell density, the astrocyte area increased in the LF group compared to the HF $(p<0.05)$ one (Figure 4E).

Microglial cells with small and rounded somas and thin, long, and highly branched processes were considered resting
A

T-maze spontaneous alternation

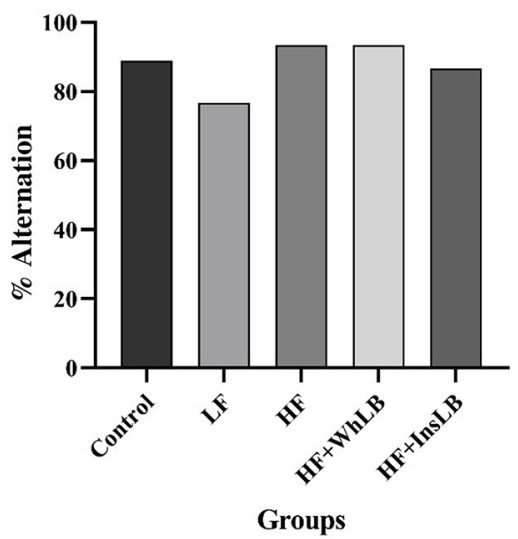

B Latency to choose

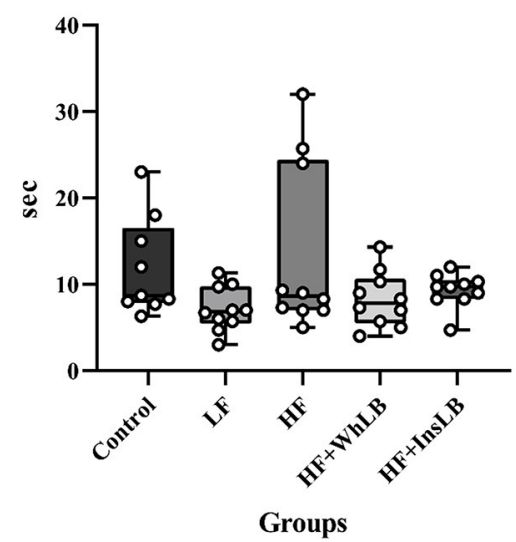

FIGURE 2 | T-maze spontaneous alternation test. (A) The percentage of alternation. (B) Latency to choose the arm in the second trial. Fisher's exact test was used for categorical data analysis. Continuous data distribution is expressed with the aid of the box and whisker plots: the median is shown by the horizontal line, the box is limited by 25th and 75th percentiles (interquartile range, IQR), while the whiskers represent the full range of data ( $n=9-10 / g r o u p)$. Comparisons among groups were performed with the non-parametric Kruskal-Wallis test, followed by Dunn's post hoc test. 
A

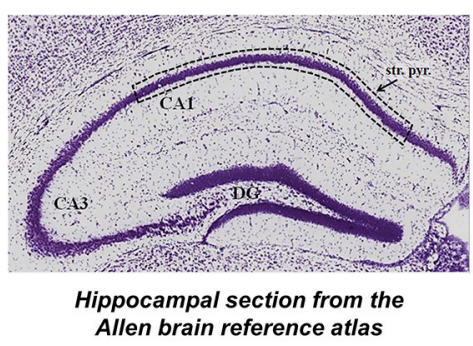

C

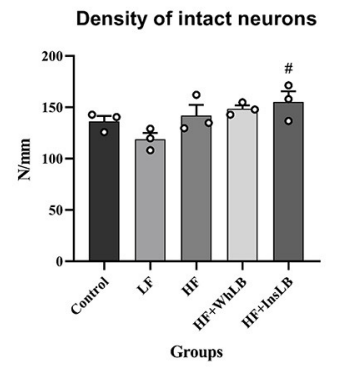

D

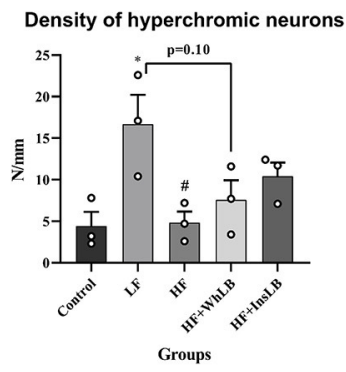

B

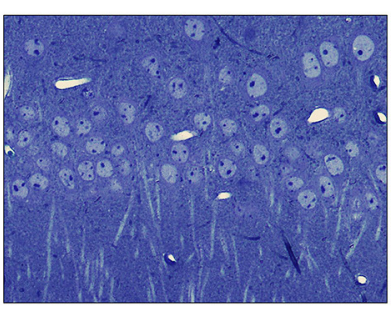

Control

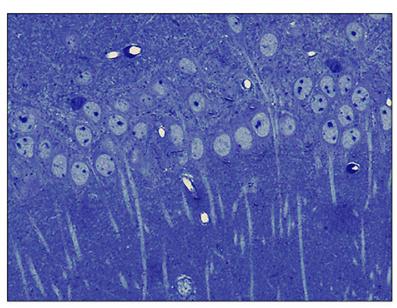

HF

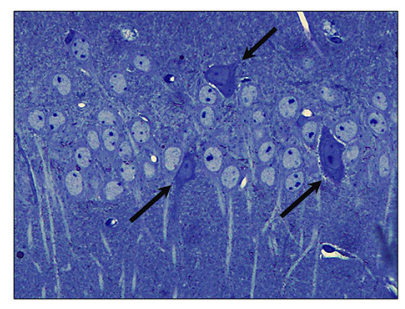

LF

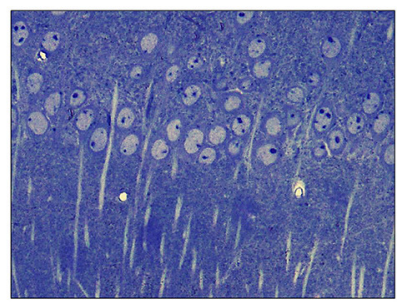

HF+WhLB

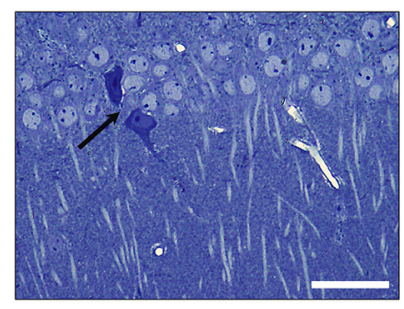

HF+InsLB

FIGURE 3 | Pyramidal neurons in the hippocampal CA1 area of ApoE-/- mice. (A) The reference image of the Nissl-stained coronal section of the dorsal hippocampus obtained from the Allen Mouse Brain Atlas. Dotted lines indicate the area of interest in the stratum pyramidale. (B) Methylene blue-stained semi-thin sections demonstrate the morphology of a pyramidal layer. Arrows point to hyperchromic damaged cells. (C) The density of intact neurons. (D) The density of hyperchromic pyramidal cells. Data are expressed as the Mean \pm SEM ( $n=3 /$ group). Comparisons among groups were performed with the one-way ANOVA, followed by Turkey's post hoc test. ${ }^{*} p<0.05$ indicates significant differences vs. control; $\# p<0.05$ indicates significant differences vs. LF. The scale bar corresponds to $20 \mu \mathrm{m}$.

microglia. An enlargement of soma with a shape change (cell body becomes bi- or tripolar, ellipsoid or rod-shaped), thickening and shortening of the base processes, and a decrease in their branching indicate the activation of microglia $(30,31)$. Most microglial cells in the hippocampal CA1 area of the control animals were characterized by small round or slightly elongated somas with thin, long, and moderately ramified processes, whereas LFD-, HFD-, and HFD+InsLB-fed ApoE-/- mice demonstrated morphological indications of microglia activation (Figures 4B,C). There were enlarged oval and, in some cases, rod-shaped somas along with both long ramified and short, poorly branched processes in the Iba-1-positive cells of LFDfed animals. Microglia with big somas and branched processes of different lengths were detected in the hippocampus of HFDfed mice. Mainly cells with small rounded or polar somas were detected in the HF+InsLB group; rod-shaped cells and signs of partial deramification were occasionally observed. The HF+WhLB group, in turn, harbored mostly morphologically resting microglia (small and predominantly rounded cell bodies and long and highly branched processes). Concerning the quantitative indicators, the number of Iba-1-positive cells per unit area was higher $(p=0.06)$ in LFD-fed ApoE-/- mice vs. those on standard chow (Figure 4F). The increase in the surface area of Iba-1-positive cells in LFD- and HFD-fed animals was substantial, by $25 \pm 3 \%(p<0.05$ and $p=0.10)$, compared to the control group. Microglial hypertrophy in the HF+WhLB and $\mathrm{HF}+\mathrm{InsLB}$ groups reduced by $25-30 \%$ relative to $\mathrm{HF}(p<0.05)$ and LF $(p<0.01)$ (Figure 4G).

\section{Ultrastructural Changes in the Hippocampal CA1 Area}

Ultrastructural analyses of the hippocampal CA1 area in different experimental groups of ApoE-/- mice (LF, HF, HF+WhLB, $\mathrm{HF}+$ InsLB) showed that the structures of their neuropil did not have critical morphological differences with those of the control animals (Figure 5A). However, some changes in the quantitative parameters of the neuropil were identified via morphometric analysis (Figures 5C-H). Control mice had $29.9 \pm 0.5$ synapses per $100 \mu \mathrm{m}^{2}$ of stratum radiatum in the hippocampal CA1 area (Figure 5C). This parameter declined nearly 1.35 times in both 


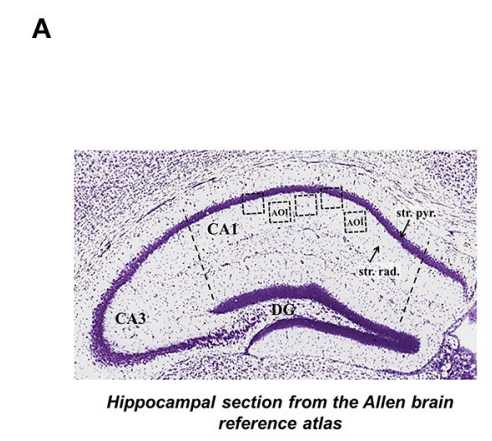

B
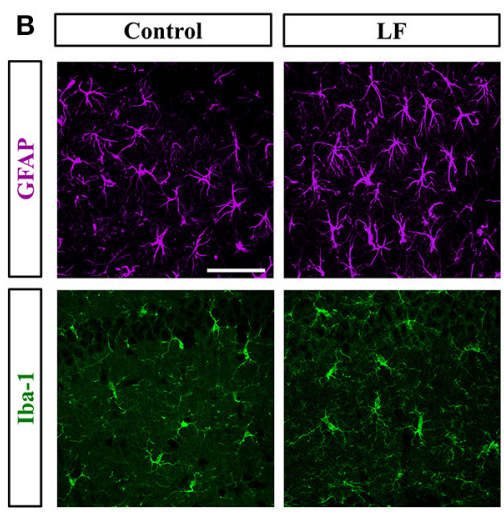

C
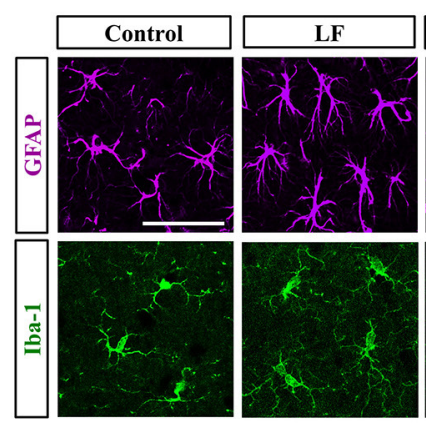
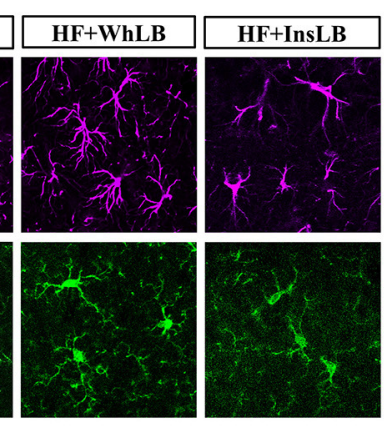
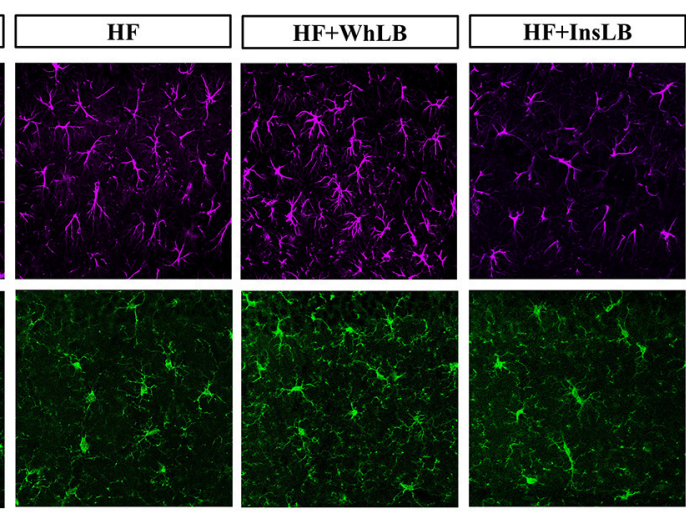

D

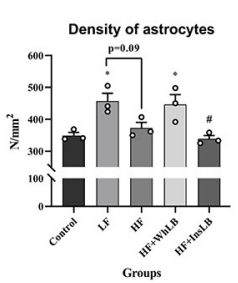

$\mathbf{F}$

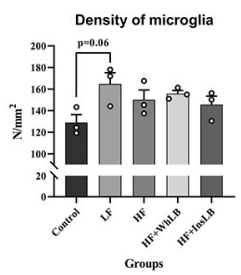

E

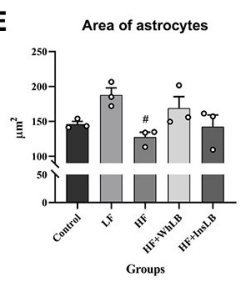

G

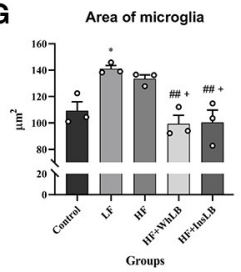

FIGURE 4 | Immunohistochemical analysis of glial cells in the hippocampal CA1 area of ApoE-/- mice. (A) The reference image of the Nissl-stained coronal section of the dorsal hippocampus obtained from the Allen Mouse Brain Atlas. Dotted line squares indicate the areas of interest (AOI) in the stratum pyramidale and stratum radiatum. (B) Immunofluorescent-labeled images of GFAP-positive astrocytes and lba-1-positive microglia at 40x objective magnification, as well as (C) cut out and zoomed fragments (crops) from these images for better visualization of the processes and somas of astrocytes and microglial cells. (D-G) The morphometric characteristics of glial cells: (D) the density of GFAP-positive astrocytes and (E) their area; (F) the density of lba-1-positive microglia and (G) the area of microglial cells. Data are expressed as the Mean \pm SEM ( $n=3$ /group). Comparisons among groups were performed with the one-way ANOVA, followed by Turkey's post hoc test. ${ }^{*} p<0.05$ indicates significant differences vs. control; ${ }^{*} p<0.05$ and $\# \# p<0.01$ indicate significant differences vs. LF; ${ }^{+} p<0.05$ indicates significant differences vs. HF. The scale bar corresponds to $50 \mu \mathrm{m}$.

the LF $(p<0.01)$ and HF $(p<0.01)$ groups compared with the control animals. Meanwhile, the inclusion of whole lingonberries and their insoluble fraction to an HFD led to an increase in synaptic density by $38 \pm 3 \%(p<0.05$ for HF+WhLB and $p$ $=0.06$ for $\mathrm{HF}+\mathrm{InsLB})$ and $42 \pm 3 \%(p<0.05)$, compared to an LFD and an HFD without berry supplements, respectively. Given that the most prevalent synaptic type is the simple one, the differences in the number of simple synapses observed among experimental groups were very similar to the differences in overall synaptic density (Figure 5D). Of note, LFD- and HFDfed mice demonstrated a dramatic decrease in the density of multiple synapses in the hippocampal CA1 area vs. control animals. The simultaneous ingestion of whole lingonberries and an HFD contributed to about a 15 -fold rise in the number of multiple synapses $(p<0.001)$ compared to the intake of an HFD (Figure 5F). Interestingly, the hippocampal synaptic density had a strong negative correlation with the area of microglial cells $(r=-0.762)$. According to the coefficient of determination $\left(r^{2}\right)$, we assume that microglia hypertrophy could cause the observed diminishing number of synapses with the likelihood of 58\% (Figure 5G). In addition, there was a statistically significant increase in PSD length (by 35-40\%) in mice on an HFD with InsLB supplementation $(p<0.05)$ relative to standard diet-, LFD-, and HFD-fed animals (Figure 5H).

\section{GFAP and NCAM in the Hippocampus}

The highest level of the filamentous form of GFAP was observed in LFD-fed mice, accounting for a concentration that was 43 , 54,31 , and $53 \%$ more than that in the control $(p<0.05)$, HF $(p<0.01), \mathrm{HF}+\mathrm{WhLB}(p=0.06)$, and HF+InsLB $(p<0.01)$ groups, respectively (Figure 6A). Meanwhile, the analysis of the soluble form of GFAP did not reveal any differences among experimental mice (Figure 6B). In addition, neither investigated diet contributed to the modulation of NCAM expression, as is evident from our data of the concentrations of its membrane and soluble forms (Figures 6C,D). 
A

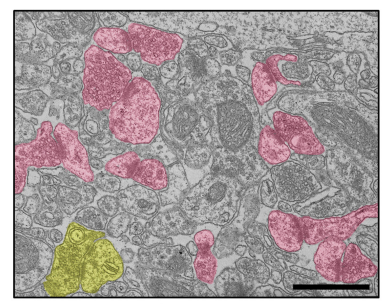

Control

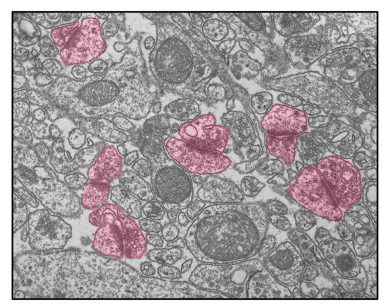

HF

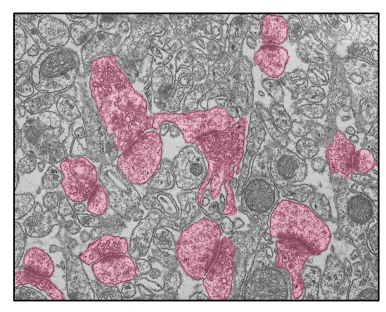

HF+InsLB

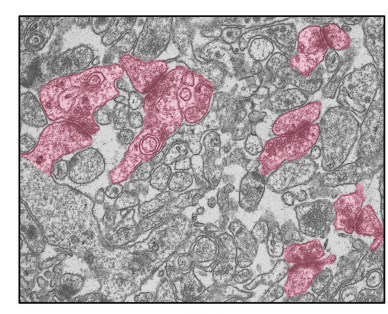

LF

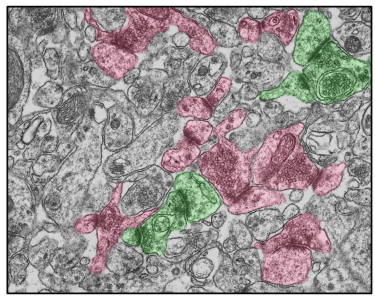

HF+WhLB

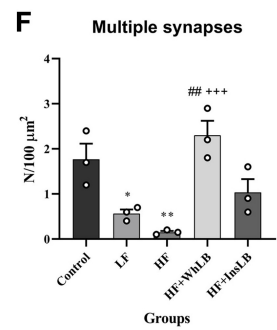

B

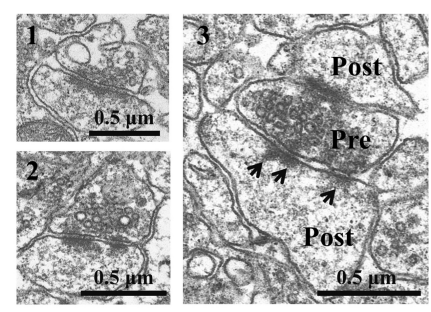

D

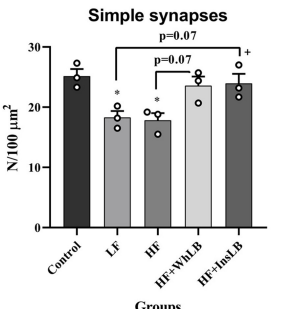

G

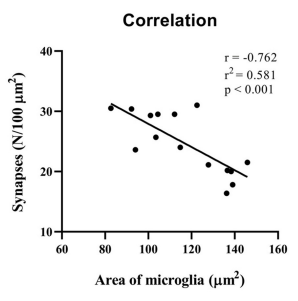

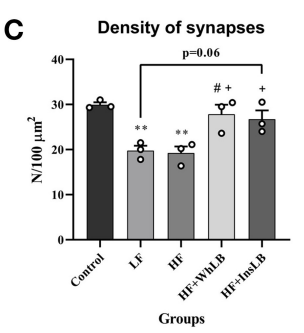

E

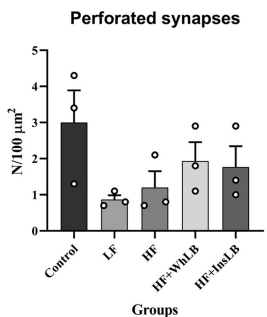

H

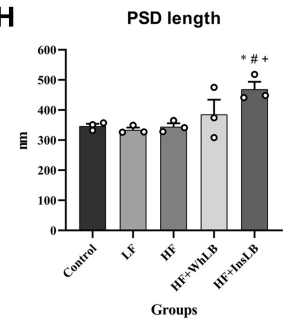

FIGURE 5 | Electron microscopic analyses of the stratum radiatum in the hippocampal CA1 area of distinct experimental groups. (A) Electron micrographs of the neuropil ultrastructure. Different types of synaptic contacts are marked in various colors, as follows: simple-pink, perforated-yellow, multiple-green. (B) Representative images of (1) simple, (2) perforated, and (3) multiple synaptic types. Pre, presynaptic terminal; Post, postsynaptic terminal: Arrows point to the postsynaptic density. (C) The density of synapses and (D) the number of simple, (E) perforated, and (F) multiple synaptic contacts per $100 \mu m^{2}$ of hippocampal neuropil. (G) Pearson's correlative analysis between the area of microglial cells and synaptic density. (H) The average length of the postsynaptic density. Data are expressed as the Mean \pm SEM ( $n=3$ /group). Comparisons among groups were performed with the one-way ANOVA, followed by Turkey's post hoc test. * $p<0.05$ and ${ }^{* \star} p<0.01$ indicate significant differences vs. control; ${ }^{\#} p<0.05$ and $\# \# p<0.01$ indicate significant differences vs. LF; ${ }^{+} p<0.05$ and ${ }^{+++} p<0.01$ indicate significant differences vs. HF. The scale bar corresponds to $1 \mu \mathrm{m}$.

\section{DISCUSSION}

It is generally believed that high-fat diets promote obesity and neuroinflammation and induce a cognitive decline in ApoE-/mice $(32,33)$. Based on this understanding, we hypothesized that an HFD intake could induce the neuroinflammation and damage of brain tissues, particularly the hippocampus, eventually leading to cognitive impairments. Lingonberries and their insoluble fraction were anticipated to prevent, at least partly, the abnormalities mentioned above (20).

In the present study, comparing the experimental diets (standard chow, an LFD, an HFD, an HFD+WhLB, and an HFD+InsLB) did not reveal their influence on short-term spatial memory in young adult ApoE-/- mice. But surprisingly, animals on an LFD, unlike other rations, demonstrated a marked increase in the density of hyperchromic (damaged) pyramidal neurons and astroglial activation (namely, elevated levels of the filamentous form of GFAP, more significant astrocyte proliferation, and their hypertrophy) in the hippocampus. Reactive astrogliosis is generally understood to enhance neuroprotection and trophic support for neurons; however, it may further lead to emerging damage and neuronal loss through the production of pro-inflammatory cytokines and ion imbalance (34). Worth noting, the indicated morphological impairments in the LF group are consistent with our previous results derived from the $16 \mathrm{~S}$ rRNA gene sequencing of cecal microbiota (unpublished data); in that study, LFD-fed mice harbored the highest Firmicutes/Bacteroidetes ratio and relatively abundant Oscillospira and Desulfovibrio among all experimental groups. Reportedly, a higher Firmicutes/Bacteroidetes ratio correlates with depression-like behavior, hippocampal astrogliosis, cognitive deficit, amyloid plaques load, obesity, and inflammation $(35,36)$, and an abundance of Oscillospira and Desulfovibrio is associated with systemic inflammation and impaired intestinal permeability $(34,37)$.

Morphological signs of astrogliosis, incidentally, were also observed in the HF+WhLB group. But, in our view, it had a different nature from that in LFD-fed mice. A possible cause of astrocyte activation in animals fed WhLB might be the high concentration of free benzoic acid in whole lingonberries (0.6-1.3 


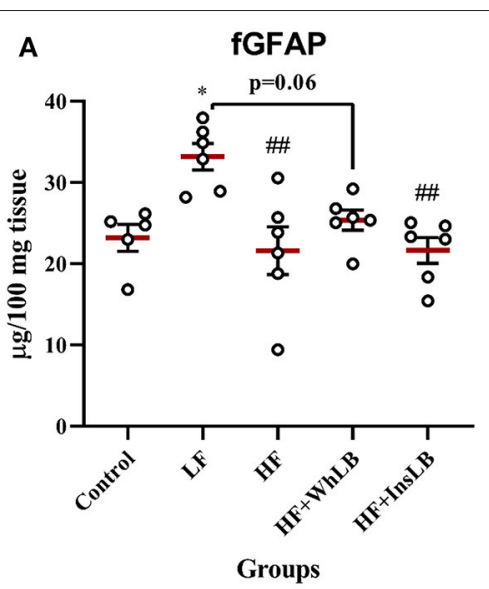

C

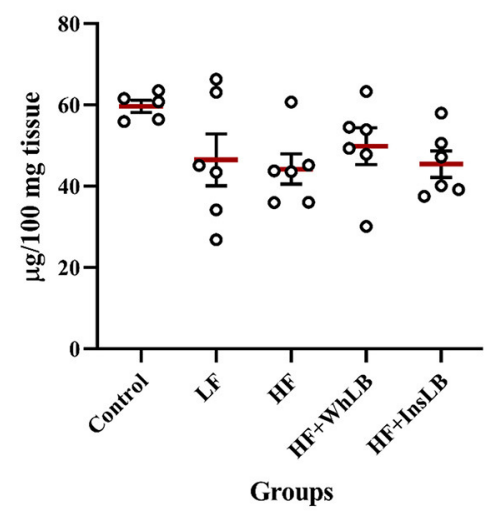

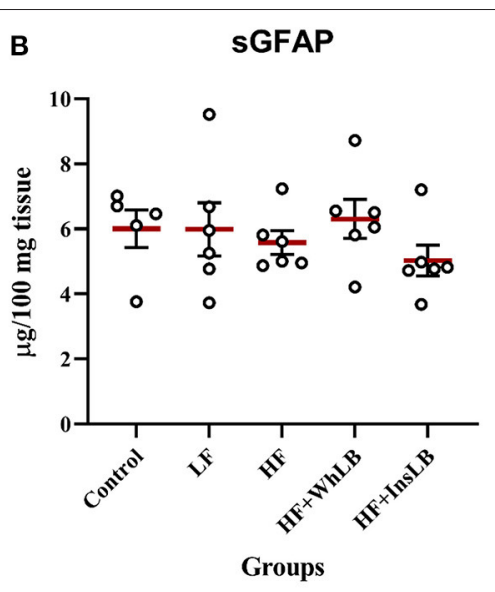

D

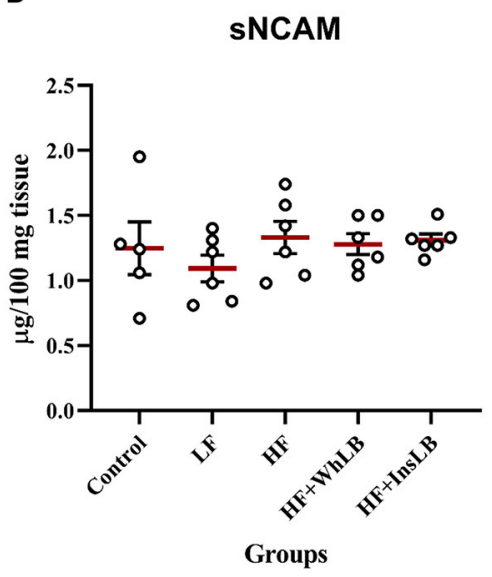

FIGURE 6 | The levels of astrocyte- and neuron-specific proteins in the hippocampus of ApoE-/- mice. The concentrations of (A) the filamentous and (B) the soluble forms of GFAP, as well as (C) the membrane and (D) the soluble forms of NCAM are presented in the form of the scatter dot plots. Data are expressed as the Mean \pm SEM ( $n=5-6$ /group). Comparisons among groups were performed with the one-way ANOVA, followed by Turkey's post hoc test. * $p<0.05$ indicates significant difference vs. control; \#\# $p<0.01$ indicates significant differences vs. LF.

$\mathrm{g} / \mathrm{L}$, about $5 \%$ of all organic acids), which is water-soluble (appears in an InsLB fraction to a lesser extent) and ensures a low $\mathrm{pH}$ in the berries ( $\mathrm{pH} 2.6-2.9)(38,39)$. Per a previous report, 80 $\mathrm{mg} / \mathrm{kg}$ bw/d of benzoic acid promotes sub-chronic oral toxicity in mice (40).

Interestingly, both LDF- and HFD-fed ApoE-/- mice had morphological signs of microglia activation (increase in the area of Iba-1-positive cells, enlargement of somas, alterations in the pattern of processes ramification in the two groups, and moderate microglia proliferation in the LF group), and a decline in hippocampal synaptic density alongside a reduction in the number of multiple synapses compared to ApoE-/animals on a regular diet and an HFD with lingonberry supplements. Many recent publications confirm the relationship between microglia on one side and synaptic plasticity and neurogenesis on the other in some physiological or pathological conditions. For instance, the hypertrophy of Iba-1-positive cells in the hippocampal CA1 area in the mouse model of Alzheimer's disease has been associated with a substantial decline in dendritic spine density (41). Furthermore, HFD-induced obesity contributes to microglial reactivity in the hippocampus of $\mathrm{C} 57 \mathrm{BL} / 6 \mathrm{~J}$ mice, as evidenced by an increase in the microglial soma area (without significant cell proliferation) along with the increased co-localization of Iba-1 and CD68 markers. In that case, microglial activation was involved in the loss of dendritic spines and synaptophysin-positive sites in the CA1 area and dentate gyrus due to microglial phagocytosis of synapses, higher expression of pro-inflammatory cytokines (TNF- $\alpha$ and IL-1 $\beta$ ), and deterioration of neurogenesis in the dentate gyrus $(42,43)$.

Considering that we observed changes in hippocampal morphology not only between the mice fed an HFD and standard chow/an LFD, but also between the intake of an LFD and standard diet, we could assume that the lipid composition of the diets was not crucial in inducing the disturbances mentioned above. As seen from the composition of the investigated diets, the most substantive quantitative and qualitative differences were noted in dietary carbohydrates (Table 1; https://sdsdiets.com/wp-content/uploads/2021/02/ 
rmlp-e-fg.pdf). The primary source of carbohydrates in the LFD and an HFD was corn starch (66 and 51 gm\%, respectively). Additionally, both diets consisted of $\sim 5.5 \mathrm{gm} \%$ of cellulose, insoluble dietary fiber. Notably, lingonberryderived fiber and other berry components substituted some starch in an HFD (34 gm\% for an HFD+WhLB and $37 \mathrm{gm} \%$ for an HFD+InsLB). Meanwhile, the standard RM-1 diet had $45 \mathrm{gm} \%$ of starch that originated from wheat, barley, wheat bran, and de-hulled extracted soya and $17 \mathrm{gm} \%$ of dietary fiber.

Generally, the more there is starch and low-molecular sugars and the less there is dietary fiber in a diet, the higher the glycemic index (GI) that diet has (44). Consequently, we can rank the diets used in the order of increasing GIs: an HFD+WhLB $\rightarrow$ an $\mathrm{HFD}+$ InsLB $\rightarrow$ standard chow $\rightarrow$ an $\mathrm{HFD} \rightarrow$ an LFD. As shown before, feeding C57BL/6J mice with high GI-diet resulted in an increased blood glucose area under the curve (AUC) during OGTT and the development of insulin resistance (45). Similarly, in our experiment, ApoE-/- mice fed an LFD and HFD demonstrated enhanced AUCs by 1624 and $10-17 \%$, respectively, vs. eating standard chow and an HFD with lingonberries (unpublished data), indicating the risk of glucose metabolism abnormalities and insulin resistance in those groups.

Some authors have pointed out an unfavorable impact of excessive dietary starch on glucose metabolism. Maekawa et al. found that a high-starch diet (starch 74\%kcal) considerably enhanced insulin secretion during OGTT and the value of HOMA-IR index in mice, a representative indication of insulin resistance (46). Patterson et al. in turn, revealed that a lowfat high-corn starch diet might cause more severe glucose intolerance in mice than a low-fat high-sucrose diet (47). However, not only total dietary starch content but its qualitative composition is relevant to glucose metabolic rate. Starch consists of two major components-amylopectin (branched and more digestible) and amylose (linear and less available for enzymatic hydrolysis). A high-amylopectin diet promotes a more significant production of postprandial blood glucose and the development of insulin resistance in rats compared to a high-amylose diet (48). Literature indicates that the amylose content in the primary plant sources of starch in the diets we applied are as follows: wheat (21.5-26.6 gm\%), barley (25.8-29.8 gm\%), and corn (20.9-25 gm\%) (49-51). Thus, heterogeneity in starch composition between standard chow and an LFD/HFD might partly contribute to morphological changes detected in the hippocampus of ApoE-/- mice, but we tend to think that the overall level of this polysaccharide plays a more critical role.

Glucose imbalance and insulin resistance correlate positively with both structural and functional impairments in the hippocampus. Diet-induced hyperglycemia in middle-aged rats has been shown to lead to disturbances in spatial learning, reduction in LTP at Shaffer collaterals, and dendritic spine density in the CA1 area (52). Diabetic mice with chronic hyperglycemia exhibit enhanced blood-brain barrier (BBB) permeability, microglia activation (Iba-1 immunoreactivity, enlarged microglial somas), and elevated TNF- $\alpha$ and IL-6 production, along with the downregulation of the expression of two synaptic markers, spinophilin and synaptophysin $(53,54)$. Moreover, chronic high blood glucose level-induced activation of microglia and neuroinflammation exacerbates apoptosis in hippocampal pyramidal neurons (55).

As mentioned before, investigated diets also varied in amount and types of dietary fiber. Standard chow was more abundant in fiber, including hemicellulose, cellulose, pectin, and lignin, than an LFD/HFD with cellulose alone. Furthermore, various ingredients of a standard diet are not equal in the content of dietary fiber; for example, total dietary fiber account for about 13, 45, and $17 \mathrm{gm} \%$ of wheat grains, wheat bran, and barley grains, respectively $(56,57)$. Barley is worth emphasizing to be rich in $\beta$-glucan $(\sim 2-$ $7 \mathrm{gm} \%)$. It has been shown that barley $\beta$-glucan reduces insulin resistance, serum glucose levels, and lipids in HFD-fed mice. Due to the modification in gut microbiota composition, barley provides an anti-inflammatory effect as well (58). Zhang et al. demonstrated that wheat fiber significantly diminishes the size of atherosclerotic plaques and, most importantly, inhibits the expression of pro-inflammatory factor NF-kB in the aorta of ApoE-/- mice (59). However, whether cereal fiber could modulate NF-kB expression in microglia, thereby impacting neuroinflammation in the hippocampus, merits detailed evaluation. In addition, dietary fiber supplements link with $\mathrm{BBB}$ integrity, hippocampal morphology, synaptogenesis, and cognitive function indirectly through the alteration of gut bacterial cenosis. Shi et al. revealed that an intake of microbiota-accessible carbohydrates (MAC) boosts the richness and $\alpha$-diversity of fecal microbiota in obese mice, especially within the Bacteroidetes taxon, compared to a fiber-deficient diet. In the hippocampus, MAC supplementation to an HFD can attenuate impairments induced by fiber-deficient ration, such as a decrease in occludin levels (tight junctional protein), reactive astrogliosis and microgliosis, the upregulation of proinflammatory cytokines (TNF- $\alpha$, IL-1 $\beta$, IL-6), the engulfment of PSD95-positive sites by microglia, and the deterioration of the recognition memory $(60,61)$.

At the ultrastructural level, we revealed that rations with lingonberries and their insoluble fraction prevented the decline in synaptic density in the CA1 stratum radiatum, observed in HFD- and LFD-fed animals. In addition, lingonberry supplements altered the ratio of synaptic types, increasing the total number of multiple synapses. Beyond that, the consumption of the insoluble fraction of the berries supported the elongation of PSD. We see such reorganization as an indication of higher structural synaptic plasticity in the hippocampus. The formation of multiple synaptic contacts is thought to enhance the efficiency of synaptic transmission and is associated with several synaptogenic stimuli, such as brain damage, LTP, hippocampus-mediated training, estrogen use, and more (62). The PSD length increase, in its turn, is linked to the increased abundance in neurotransmitter receptors on a postsynaptic membrane and, as a result, more intensive neurotransmission (63). Interestingly, the enhanced hippocampal synaptic density in the HF+WhLB and HF+InsLB groups correlated with the decrease in microglial cellular 
area. Thus, in our study, microglial activation may serve as a mechanism of structural synaptic plasticity regulation. The synaptic improvements in the hippocampi of animals fed lingonberries and their insoluble fraction were also accompanied by a substantial rise in the relative abundance of Akkermansia in the cecum, as well as a decrease in Mucispirillum, unlike in the HF group (19). Mucispirillum is associated with gastrointestinal inflammation and age-related changes (64), whereas, Akkermansia normalizes lipid profile and blood glucose levels during obesity, attenuates $A \beta$ pathology, restores neuronal development and synaptic plasticity, and provides antiinflammatory effects (65-67).

The growth and maintenance of beneficial gut microbiota in ApoE-/- mice could be achieved through dietary fiber and phenolic compounds (e.g., quercetin, catechin, resveratrol, anthocyanins, and more), which are abundant in lingonberries (68). For example, a blackberry anthocyanin-rich extract has shown a neuroprotective effect in rats fed an HFD by modulating the gut microbiota and enhancing kynurenic acid production (a neuroprotective agent) (69). Combining quercetin with resveratrol restores HFD-induced intestinal microbiota dysbiosis in rats and increases the relative abundance of Akkermansia muciniphila (70).

Regarding the role of dietary fats, in our study, their excess in an HFD had a neutral effect on the hippocampal structure of ApoE-/- mice rather than a negative one. As illustrated in Table 1, all fats in standard chow and LFD constituted soybean oil, whereas an HFD contains not only soybean oil but also lard, which is rich in saturated (39$45 \%)$ and monounsaturated (42-45\%) fatty acids (71, 72). Brain phospholipids have recently been shown to incorporate monounsaturated fatty acids more frequently than phospholipids in rodent heart, liver, and kidney (73). Thus, we do not exclude the potential impact of a lard-rich HFD on neuronal plasticity maintenance in young ApoE-/- mice. Besides, the 8-week feeding period might be insufficient for noticeable hippocampal and behavioral effects of an HFD to develop. Therefore, further investigations must be carried out to scrutinize this hypothesis.

Since different dietary exposures on animals with ApoE deficiency have being studied for more than a couple of decades, many divergent observations have accumulated. Their comparison is complicated by variations between experiments such as rodent's age and sex, composition of diets, duration of feeding, etc. For instance, Mulder et al. showed a dramatically increased $\mathrm{BBB}$ permeability in adult ApoE-/- mice with chronic HFD intake (74), whereas Bai et al. demonstrated that the consumption of an HFD elevated levels of GFAP, pro-inflammatory and pro-apoptotic factors in the cortex and hippocampus of 20-week-old male ApoE-/- mice (75). Another work suggests that 5 -week feeding of ApoE-deficient rats with an HFD upregulated IL-1 $\beta$ and decreased the expression of occludin in the brain (signs of neuroinflammatory reaction), compared with an LFD (76). In contrast, there is evidence indicating the beneficial effect of a high-fat/high-cholesterol diet on the expression of BDNF and TrkB mRNAs in the hippocampus of 20-week-old ApoE-/- mice, which is related to improved synaptic plasticity (77). Meanwhile, our morphological and behavioral data are largely consistent with certain previously published papers. Crisby et al. also found microgliosis and no difference in the density of GFAP-positive astrocytes in the hippocampus of adult ApoE-/- mice fed an HFD, as compared to those on a standard diet (78). Other researchers, in turn, state that an HFD did not affect the spatial memory of middle-aged ApoE-/- females (4). In addition, the obtained results on the impact of lingonberries on the structural synaptic plasticity complement existing information concerning the ability of bioactive compounds from fruits and berries to correct the metabolic and neurological alterations in ApoE-deficient mice including reduction of the atherosclerotic lesion (20), enhancement of antioxidant protection (79), increasing the level of brain acetylcholine (80), and so on.

It is worth pointing out several limitations when interpreting and extrapolating the present results. Firstly, only males were examined in our study that constrains the spreading of the findings on the entire population of ApoE-/mice. Secondly, due to the relatively young age of the investigated animals and thereby insufficiently marked neuromorphological and cognitive alterations, a favorable or adverse impact of the diets may not be fully manifested. Thirdly, additional research on wild-type mice appears to us would enable more comprehensively interpret the obtained data, as well as explores possible outcomes from the consumption of various dietary components, depending on ApoE genotype.

In summary, we revealed that ApoE-/- mice on an LFD were characterized by the hyperchromatosis of the pyramidal neurons and signs of neuroinflammation in the hippocampal CA1 area. By contrast, the consumption of an HFD did not substantially negatively affect the cellular structure of the hippocampus. However, both diets contributed to synaptic loss along with a shift in the number of multiple synapses and moderate microglia hypertrophy. Obtained results may be related not so much with the fat content of diets used but rather with the composition of carbohydrates. Our study demonstrates that reducing the starch amount and increasing dietary fiber in a ration is more favorable for maintaining the healthy hippocampal structure in young adult male ApoE-/- mice. The inclusion of lingonberries and their insoluble fraction to an HFD seems to provide a neuroprotective effect on the altered structural synaptic plasticity. Meanwhile, the above-mentioned morphological changes in the hippocampus of ApoE-/- mice did not escalate into severe spatial memory impairments.

\section{DATA AVAILABILITY STATEMENT}

The datasets generated for this study are available on request to the corresponding author. 


\section{ETHICS STATEMENT}

The animal study was reviewed and approved by the Local Ethical Committee for Animal Experiments at Lund University, Sweden.

\section{AUTHOR CONTRIBUTIONS}

GS, OP, FH, and NM designed the study. TK, IO, NM, and GU performed the experiment, sample collection, and behavioral testing. GU and DM carried out ELISA. DS, TK, IO, KS, and GS conducted morphological analyses and interpreted results. DS wrote the manuscript. All authors revised and approved the final version of the manuscript.

\section{REFERENCES}

1. Jankowsky JL, Zheng $H$. Practical considerations for choosing a mouse model of Alzheimer's disease. Mol Neurodegener. (2017) 12:89. doi: 10.1186/s13024-017-0231-7

2. Mahley RW. Central nervous system lipoproteins: ApoE and regulation of cholesterol metabolism. Arterioscler Thromb Vasc Biol. (2016) 36:130515. doi: 10.1161/ATVBAHA.116.307023

3. Vance JE. Dysregulation of cholesterol balance in the brain: contribution to neurodegenerative diseases. Dis Model Mech. (2012) 5:746-55. doi: 10.1242/dmm.010124

4. Janssen CIF, Jansen D, Mutsaers MPC, Dederen PJWC, Geenen B, Mulder MT, et al. The effect of a high-fat diet on brain plasticity, inflammation and cognition in female ApoE4-knockin and ApoE-knockout mice. PloS ONE. (2016) 11:e0155307. doi: 10.1371/journal.pone.0155307

5. Fuentes D, Fernández N, García Y, García T, Morales AR, Menéndez R. Agerelated changes in the behavior of apolipoprotein E knockout mice. Behav Sci Basel Switz. (2018) 8:33. doi: 10.3390/bs8030033

6. Lo Sasso G, Schlage WK, Boué S, Veljkovic E, Peitsch MC, Hoeng J. The Apoe(-/-) mouse model: a suitable model to study cardiovascular and respiratory diseases in the context of cigarette smoke exposure and harm reduction. J Transl Med. (2016) 14:146. doi: 10.1186/s12967-016-0901-1

7. Zhang SH, Reddick RL, Piedrahita JA, Maeda N. Spontaneous hypercholesterolemia and arterial lesions in mice lacking apolipoprotein E. Science. (1992) 258:468-71. doi: 10.1126/science.1411543

8. Chapman S, Michaelson DM. Specific neurochemical derangements of brain projecting neurons in apolipoprotein E-deficient mice. J Neurochem. (1998) 70:708-14. doi: 10.1046/j.1471-4159.1998.70020708.x

9. Gordon I, Genis I, Grauer E, Sehayek E, Michaelson DM. Biochemical and cognitive studies of apolipoprotein-E-deficient mice. Mol Chem Neuropathol. (1996) 28:97-103. doi: 10.1007/BF02815210

10. Masliah E, Mallory M, Ge N, Alford M, Veinbergs I, Roses AD. Neurodegeneration in the central nervous system of apoE-deficient mice. Exp Neurol. (1995) 136:107-22. doi: 10.1006/exnr.1995.1088

11. Zhao X-S, Wu Q, Peng J, Pan L-H, Ren Z, Liu H-T, et al. Hyperlipidemiainduced apoptosis of hippocampal neurons in apoE(-/-) mice may be associated with increased PCSK9 expression. Mol Med Rep. (2017) 15:71218. doi: 10.3892/mmr.2016.6055

12. Guaraldi M, Shea TB. A high-fat and high-cholesterol diet potentiates oxidative damage in hippocampus of mice lacking apolipoprotein E. Open Neurol J. (2018) 12:12-18. doi: 10.2174/1874205X01812010012

13. Famer D, Wahlund L-O, Crisby M. Rosuvastatin reduces microglia in the brain of wild type and ApoE knockout mice on a high cholesterol diet; implications for prevention of stroke and AD. Biochem Biophys Res Commun. (2010) 402:367-72. doi: 10.1016/j.bbrc.2010.10.035

14. Rühlmann C, Wölk T, Blümel T, Stahn L, Vollmar B, Kuhla A. Long-term caloric restriction in ApoE-deficient mice results in neuroprotection via Fgf21-induced AMPK/mTOR pathway. Aging. (2016) 8:2777-89. doi: 10.18632/aging.101086

\section{FUNDING}

This study was funded by the Direktör Albert Påhlsson Foundation Grant (No. 2019-169) and the Swedish Research Council FORMAS Grant (No. 2015-00877).

\section{ACKNOWLEDGMENTS}

The authors thank Camilla Björklöv and Agnieszka Czopek for help with animal maintenance, and Honore N. Shiyntum for the proofreading of the manuscript.

15. Vorhees CV, Williams MT. Morris water maze: procedures for assessing spatial and related forms of learning and memory. Nat Protoc. (2006) 1:84858. doi: 10.1038/nprot.2006.116

16. Kelly E, Vyas P, Weber JT. Biochemical properties and neuroprotective effects of compounds in various species of berries. Mol Basel Switz. (2017) 23:26. doi: 10.3390/molecules23010026

17. Isaak CK, Petkau JC, O K, Debnath SC, Siow YL. Manitoba lingonberry (Vaccinium vitis-idaea) bioactivities in ischemia-reperfusion injury. J Agric Food Chem. (2015) 63:5660-9. doi: 10.1021/acs.jafc.5b00797

18. Pacheco SM, Azambuja JH, de Carvalho TR, Soares MSP, Oliveira PS, da Silveira EF, et al. Glioprotective effects of lingonberry extract against altered cellular viability, acetylcholinesterase activity, and oxidative stress in lipopolysaccharide-treated astrocytes. Cell Mol Neurobiol. (2018) 38:110721. doi: 10.1007/s10571-018-0581-x

19. Marungruang N, Kovalenko T, Osadchenko I, Voss U, Huang F, Burleigh $S$, et al. Lingonberries and their two separated fractions differently alter the gut microbiota, improve metabolic functions, reduce gut inflammatory properties, and improve brain function in ApoE-/- mice fed highfat diet. Nutr Neurosci. (2018) 23:600-12. doi: 10.1080/1028415X.2018.15 36423

20. Matziouridou C, Marungruang N, Nguyen TD, Nyman M, Fåk F. Lingonberries reduce atherosclerosis in Apoe(-/-) mice in association with altered gut microbiota composition and improved lipid profile. Mol Nutr Food Res. (2016) 60:1150-60. doi: 10.1002/mnfr.201500738

21. Deacon RMJ, Rawlins JNP. T-maze alternation in the rodent. Nat Protoc. (2006) 1:7-12. doi: 10.1038/nprot.2006.2

22. Lein ES, Hawrylycz MJ, Ao N, Ayres M, Bensinger A, Bernard A, et al. Genome-wide atlas of gene expression in the adult mouse brain. Nature. (2007) 445:168-76. doi: 10.1038/nature05453

23. Kovalenko TN, Ushakova GA, Osadchenko I, Skibo GG, Pierzynowski SG. The neuroprotective effect of 2-oxoglutarate in the experimental ischemia of hippocampus. J Physiol Pharmacol. (2011) 62:239-46. Available online at: https://www.jpp.krakow.pl/journal/archive/04_11/pdf/239_04_11_article.pdf

24. Weakley BS. A Beginner's Handbook in Biological Electron Microscopy. Edinburgh: Churchill Livingstone (1972). p. 228.

25. Kirino T, Tamura A, Sano K. Selective vulnerability of the hippocampus to ischemia - reversible and irreversible types of ischemic cell damage. Prog Brain Res. (1985) 63:39-58.

26. Fomenko OZ, Ushakova HO, Piierzhynovs'kyi SH. [Astroglia proteins in the rat brain in experimental chronic hepatitis and 2-oxoglutarate effect]. Ukr Biokhimichnyi Zhurnal. (2011) 83:69-76. Available online at: http://ubj. biochemistry.org.ua/images/stories/pdf/2011/UBJ_N1_2011/Fomenko_83_1. pdf

27. Choi M, Ahn S, Yang E-J, Kim H, Chong YH, Kim H-S. Hippocampus-based contextual memory alters the morphological characteristics of astrocytes in the dentate gyrus. Mol Brain. (2016) 9:72. doi: 10.1186/s13041-0160253-z

28. Sun D, Jakobs TC. Structural remodeling of astrocytes in the injured CNS Neuroscientist. (2012) 18:567-88. doi: 10.1177/1073858411423441 
29. Oberheim NA, Goldman SA, Nedergaard M. Heterogeneity of astrocytic form and function. In: Milner R, editor. Astrocytes. Methods in Molecular Biology. Totowa, NJ: Humana Press (2012). p. 23-45.

30. Cerbai F, Lana D, Nosi D, Petkova-Kirova P, Zecchi S, Brothers HM, et al. The neuron-astrocyte-microglia triad in normal brain ageing and in a model of neuroinflammation in the rat hippocampus. PLoS ONE. (2012) 7:e45250. doi: 10.1371/journal.pone.0045250

31. Miller KR, Streit WJ. The effects of aging, injury and disease on microglial function: a case for cellular senescence. Neuron Glia Biol. (2007) 3:24553. doi: 10.1017/S1740925X08000136

32. Bink D. Neuropathological Changes in Mouse Models of Cardiovascular Diseases(2016). Available online at: http://hdl.handle.net/11245/1.534716 (accessed May 13, 2020).

33. Park Y-J, Ko JW, Jeon S, Kwon YH. Protective effect of genistein against neuronal degeneration in ApoE-/- mice fed a high-fat diet. Nutrients. (2016) 8:692. doi: 10.3390/nu8110692

34. Thevaranjan N, Puchta A, Schulz C, Naidoo A, Szamosi JC, Verschoor CP, et al. Age-Associated microbial dysbiosis promotes intestinal permeability, systemic inflammation, and macrophage dysfunction. Cell Host Microbe. (2017) 21:455-66.e4. doi: 10.1016/j.chom.2017.03.002

35. Hassan AM, Mancano G, Kashofer K, Fröhlich EE, Matak A, Mayerhofer $\mathrm{R}$, et al. High-fat diet induces depression-like behaviour in mice associated with changes in microbiome, neuropeptide Y, and brain metabolome. Nutr Neurosci. (2019) 22:877-93. doi: 10.1080/1028415X.2018.1465713

36. Wang S, Jiang W, Ouyang $\mathrm{T}$, Shen X-Y, Wang F, Qu Y-H, et al. Jatrorrhizine balances the gut microbiota and reverses learning and memory deficits in APP/PS1 transgenic mice. Sci Rep. (2019) 9:19575. doi: 10.1038/s41598-019-56149-9

37. Terzo S, Mulè F, Caldara GF, Baldassano S, Puleio R, Vitale M, et al. Pistachio consumption alleviates inflammation and improves gut microbiota composition in mice fed a high-fat diet. Int J Mol Sci. (2020) 21:365. doi: 10.3390/ijms21010365

38. Viljakainen S, Laakso S. Acidity reduction in northern region berry juices by the malolactic bacterium Oenococcus oeni. Eur Food Res Technol. (2002) 214:412-17. doi: 10.1007/s00217-001-0470-1

39. Visti A, Viljakainen S, Laakso S. Preparation of fermentable lingonberry juice through removal of benzoic acid by Saccharomyces cerevisiae yeast. Food Res Int. (2003) 36:597-602. doi: 10.1016/S0963-9969(03)00007-3

40. EFSA ANS Panel. Scientific Opinion on the re-evaluation of benzoic acid (E 210), sodium benzoate (E 211), potassium benzoate (E 212) and calcium benzoate (E 213) as food additives. EFSA J. (2016) 14:110. doi: 10.2903/j.efsa.2016.4433

41. Pedrazzoli M, Losurdo M, Paolone G, Medelin M, Jaupaj L, Cisterna B, et al. Glucocorticoid receptors modulate dendritic spine plasticity and microglia activity in an animal model of Alzheimer's disease. Neurobiol Dis. (2019) 132:104568. doi: 10.1016/j.nbd.2019.104568

42. Cope EC, LaMarca EA, Monari PK, Olson LB, Martinez S, Zych $\mathrm{AD}$, et al. Microglia play an active role in obesity-associated cognitive decline. J Neurosci. (2018) 38:8889-904. doi: 10.1523/JNEUROSCI.0789-1 8.2018

43. Vinuesa A, Bentivegna M, Calfa G, Filipello F, Pomilio C, Bonaventura MM, et al. Early exposure to a high-fat diet impacts on hippocampal plasticity: implication of microglia-derived exosome-like extracellular vesicles. Mol Neurobiol. (2019) 56:5075-94. doi: 10.1007/s12035-018-1435-8

44. Karathanasis SK, Schiebinger RJ. Drug treatment in the metabolic syndrome. In: Rios MS, Caro JF, Carraro R, Fuentes JAG, editors. The Metabolic Syndrome at the Beginning of the XXI Century. Madrid: Elsevier (2005). p. 431-61. doi: 10.1016/B978-84-8174-892-5.50027-9

45. Isken F, Klaus S, Petzke KJ, Loddenkemper C, Pfeiffer AFH, Weickert MO. Impairment of fat oxidation under high- vs. low-glycemic index diet occurs before the development of an obese phenotype. Am J Physiol Endocrinol Metab. (2010) 298:E287-95. doi: 10.1152/ajpendo.00515.2009

46. Maekawa R, Ogata H, Murase M, Harada N, Suzuki K, Joo E, et al. Glucosedependent insulinotropic polypeptide is required for moderate high-fat dietbut not high-carbohydrate diet-induced weight gain. Am J Physiol Endocrinol Metab. (2018) 314:E572-83. doi: 10.1152/ajpendo.00352.2017

47. Patterson E, O' Doherty RM, Murphy EF, Wall R, O’ Sullivan O, Nilaweera K, et al. Impact of dietary fatty acids on metabolic activity and host intestinal microbiota composition in C57BL/6J mice. Br J Nutr. (2014) 111:190517. doi: 10.1017/S0007114514000117

48. Byrnes SE, Miller JC, Denyer GS. Amylopectin starch promotes the development of insulin resistance in rats. J Nutr. (1995) 125:1430-7.

49. Cornejo-Ramírez YI, Martínez-Cruz O, Del Toro-Sánchez CL, Wong-Corral FJ, Borboa-Flores J, Cinco-Moroyoqui FJ. The structural characteristics of starches and their functional properties. CyTA J Food. (2018) 16:100317. doi: 10.1080/19476337.2018.1518343

50. Asare EK, Jaiswal S, Maley J, Båga M, Sammynaiken R, Rossnagel BG, et al. Barley grain constituents, starch composition, and structure affect starch in vitro enzymatic hydrolysis. J Agric Food Chem. (2011) 59:474354. doi: 10.1021/jf200054e

51. Hallauer AR, editor. Specialty Corns. 2 ed. Boca Raton, FL: CRC Press (2001). p. 479.

52. Stranahan AM, Norman ED, Lee K, Cutler RG, Telljohann RS, Egan $\mathrm{JM}$, et al. Diet-induced insulin resistance impairs hippocampal synaptic plasticity and cognition in middle-aged rats. Hippocampus. (2008) 18:10858. doi: 10.1002/hipo. 20470

53. Han R, Liu Z, Sun N, Liu S, Li L, Shen Y, et al. BDNF alleviates neuroinflammation in the hippocampus of type 1 diabetic mice via blocking the aberrant HMGB1/RAGE/NF-кB pathway. Aging Dis. (2019) 10:61125. doi: 10.14336/AD.2018.0707

54. Rom S, Zuluaga-Ramirez V, Gajghate S, Seliga A, Winfield M, Heldt NA, et al. Hyperglycemia-driven neuroinflammation compromises BBB leading to memory loss in both Diabetes Mellitus (DM) type 1 and type 2 mouse models. Mol Neurobiol. (2019) 56:1883-96. doi: 10.1007/s12035-018-1195-5

55. Yun J, Lee D, Jeong H, Kim HS, Ye S, Cho C. STAT3 activation in microglia exacerbates hippocampal neuronal apoptosis in diabetic brains. J Cell Physiol. (2021) 236:7058-70. doi: 10.1002/jcp.30373

56. Fardet A. New hypotheses for the health-protective mechanisms of whole-grain cereals: what is beyond fibre? Nutr Res Rev. (2010) 23:65134. doi: 10.1017/S0954422410000041

57. Bader Ul Ain H, Saeed F, Khan MA, Niaz B, Rohi M, Nasir MA, et al. Modification of barley dietary fiber through thermal treatments. Food Sci Nutr. (2019) 7:1816-20. doi: 10.1002/fsn3.1026

58. Zeng Y, Pu X, Du J, Yang X, Li X, Mandal MdSN, et al. Molecular mechanism of functional ingredients in barley to combat human chronic diseases. Oxid Med Cell Longev. (2020) 2020:1-26. doi: 10.1155/2020/3836172

59. Zhang R, Han S, Zhang Z, Zhang W, Yang J, Wan Z, et al. Cereal fiber ameliorates high-fat/cholesterol-diet-induced atherosclerosis by modulating the NLRP3 inflammasome pathway in ApoE -/- Mice. J Agric Food Chem. (2018) 66:4827-34. doi: 10.1021/acs.jafc.8b00380

60. Shi H, Wang Q, Zheng M, Hao S, Lum JS, Chen X, et al. Supplement of microbiota-accessible carbohydrates prevents neuroinflammation and cognitive decline by improving the gut microbiota-brain axis in diet-induced obese mice. J Neuroinflammation. (2020) 17:77. doi: 10.1186/s12974-020-01760-1

61. Shi $\mathrm{H}$, Yu Y, Lin $\mathrm{D}$, Zheng $\mathrm{P}$, Zhang $\mathrm{P}, \mathrm{Hu} \mathrm{M}$, et al. $\beta$-glucan attenuates cognitive impairment via the gut-brain axis in diet-induced obese mice. Microbiome. (2020) 8:143. doi: 10.1186/s40168-020-00920-y

62. Reilly JE, Hanson HH, Fernández-Monreal M, Wearne SL, Hof PR, Phillips GR. Characterization of MSB synapses in dissociated hippocampal culture with simultaneous pre- and postsynaptic live microscopy. PloS ONE. (2011) 6:e26478. doi: 10.1371/journal.pone.0026478

63. Sheng M, Kim E. The postsynaptic organization of synapses. Cold Spring Harb Perspect Biol. (2011) 3:a005678. doi: 10.1101/cshperspect.a005678

64. Shenghua P, Ziqin Z, Shuyu T, Huixia Z, Xianglu R, Jiao G. An integrated fecal microbiome and metabolome in the aged mice reveal anti-aging effects from the intestines and biochemical mechanism of FuFang zhenshu TiaoZhi(FTZ). Biomed Pharmacother Biomedecine Pharmacother. (2020) 121:109421. doi: 10.1016/j.biopha.2019.109421

65. Dao MC, Everard A, Aron-Wisnewsky J, Sokolovska N, Prifti E, Verger EO, et al. Akkermansia muciniphila and improved metabolic health during a dietary intervention in obesity: relationship with gut microbiome richness and ecology. Gut. (2016) 65:426-36. doi: 10.1136/gutjnl-2014-308778

66. Li Z, Zhu H, Zhang L, Qin C. The intestinal microbiome and Alzheimer's disease: a review. Anim Models Exp Med. (2018) 1:180-8. doi: 10.1002/ame2.12033 
67. Yang $\mathrm{Y}$, Zhong Z, Wang $\mathrm{B}$, Xia X, Yao W, Huang $\mathrm{L}$, et al. Early-life high-fat diet-induced obesity programs hippocampal development and cognitive functions via regulation of gut commensal Akkermansia muciniphila. Neuropsychopharmacology. (2019) 44:2054-64. doi: 10.1038/s41386-019-0437-1

68. Bujor O-C. Extraction, Identification and Antioxidant Activity of the Phenolic Secondary Metabolites Isolated From the Leaves, Stems and Fruits of Two Shrubs of the Ericaceae Family. Université d'Avignon. Available online at: https://tel.archives-ouvertes.fr/tel-01722698/document (2016). p. 252.

69. Marques C, Fernandes I, Meireles M, Faria A, Spencer JPE, Mateus N, et al. Gut microbiota modulation accounts for the neuroprotective properties of anthocyanins. Sci Rep. (2018) 8:11341. doi: 10.1038/s41598-018-29744-5

70. Zhao L, Zhang Q, Ma W, Tian F, Shen H, Zhou M. A combination of quercetin and resveratrol reduces obesity in high-fat diet-fed rats by modulation of gut microbiota. Food Funct. (2017) 8:4644-56. doi: 10.1039/C7FO01383C

71. da Silva RC, Soares FASDM, Fernandes TG, Castells ALD, da Silva KCG, Gonçalves MIA, et al. Interesterification of lard and soybean oil blends catalyzed by immobilized lipase in a continuous packed bed reactor. J Am Oil Chem Soc. (2011) 88:1925-33. doi: 10.1007/s11746-011-1869-x

72. Ferrucci D, Silva SP, Rocha A, Nascimento L, Vieira AS, Taboga SR, et al. Dietary fatty acid quality affects systemic parameters and promotes prostatitis and pre-neoplastic lesions. Sci Rep. (2019) 9:19233. doi: 10.1038/s41598-019-55882-5

73. Choi J, Yin T, Shinozaki K, Lampe JW, Stevens JF, Becker LB, et al. Comprehensive analysis of phospholipids in the brain, heart, kidney, and liver: brain phospholipids are least enriched with polyunsaturated fatty acids. Mol Cell Biochem. (2018) 442:187-201. doi: 10.1007/s11010-017-3203-x

74. Mulder M, Blokland A, van den Berg D-J, Schulten H, Bakker AHF, Terwel D, et al. Apolipoprotein E protects against neuropathology induced by a high-fat diet and maintains the integrity of the blood-brain barrier during aging. Lab Invest. (2001) 81:953-60. doi: 10.1038/labinvest.3780307

75. Bai Y, Feng Y, Jiang B, Yang Y, Pei Z, Yang Q, et al. The role of exercise in reducing hyperlipidemia-induced neuronal damage in apolipoprotein $\mathrm{E}$ deficient mice. BioMed Res Int. (2021) 2021:1-9. doi: 10.1155/2021/5512518

76. Nguyen TD, Hållenius FF, Lin X, Nyman M, Prykhodko O. Monobutyrin and monovalerin affect brain short-chain fatty acid profiles and tight-junction protein expression in ApoE-knockout rats fed high-fat diets. Nutrients. (2020) 12:1202. doi: 10.3390/nu12041202

77. Wang Z-Y, Miki T, Ding Y, Wang S-J, Gao Y-H, Wang X-L, et al. A high cholesterol diet given to apolipoprotein E-knockout mice has a differential effect on the various neurotrophin systems in the hippocampus. Metab Brain Dis. (2011) 26:185-94. doi: 10.1007/s11011-011-9252-Z

78. Crisby M, Rahman SMA, Sylvén C, Winblad B, Schultzberg M. Effects of high cholesterol diet on gliosis in apolipoprotein E knockout mice. Implications for Alzheimer's disease and stroke. Neurosci Lett. (2004) 369:8792. doi: 10.1016/j.neulet.2004.05.057

79. Wu X, Kang J, Xie C, Burris R, Ferguson ME, Badger TM, et al. Dietary blueberries attenuate atherosclerosis in apolipoprotein E-deficient mice by upregulating antioxidant enzyme expression. J Nutr. (2010) 140:162832. doi: 10.3945/jn.110.123927

80. Chan A, Graves V, Shea TB. Apple juice concentrate maintains acetylcholine levels following dietary compromise. J Alzheimers Dis JAD. (2006) 9:28791. doi: 10.3233/JAD-2006-9308

Conflict of Interest: The authors declare that the research was conducted in the absence of any commercial or financial relationships that could be construed as a potential conflict of interest.

Publisher's Note: All claims expressed in this article are solely those of the authors and do not necessarily represent those of their affiliated organizations, or those of the publisher, the editors and the reviewers. Any product that may be evaluated in this article, or claim that may be made by its manufacturer, is not guaranteed or endorsed by the publisher.

Copyright (c) 2022 Shepilov, Kovalenko, Osadchenko, Smozhanyk, Marungruang, Ushakova, Muraviova, Hållenius, Prykhodko and Skibo. This is an open-access article distributed under the terms of the Creative Commons Attribution License (CC $B Y)$. The use, distribution or reproduction in other forums is permitted, provided the original author(s) and the copyright owner(s) are credited and that the original publication in this journal is cited, in accordance with accepted academic practice. No use, distribution or reproduction is permitted which does not comply with these terms. 